\title{
Integrated multilevel image fusion and match score fusion of visible and infrared face images for robust face recognition
}

\author{
Richa Singh, Mayank Vatsa, Afzel Noore* \\ Lane Department of Computer Science and Electrical Engineering, West Virginia University, USA
}

Received 1 May 2007; accepted 26 June 2007

\begin{abstract}
This paper presents an integrated image fusion and match score fusion of multispectral face images. The fusion of visible and long wave infrared face images is performed using $2 v$-granular SVM which uses multiple SVMs to learn both the local and global properties of the multispectral face images at different granularity levels and resolution. The $2 v$-GSVM performs accurate classification which is subsequently used to dynamically compute the weights of visible and infrared images for generating a fused face image. 2D log polar Gabor transform and local binary pattern feature extraction algorithms are applied to the fused face image to extract global and local facial features, respectively. The corresponding match scores are fused using Dezert Smarandache theory of fusion which is based on plausible and paradoxical reasoning. The efficacy of the proposed algorithm is validated using the Notre Dame and Equinox databases and is compared with existing statistical, learning, and evidence theory based fusion algorithms.
\end{abstract}

(C) 2007 Pattern Recognition Society. Published by Elsevier Ltd. All rights reserved.

Keywords: Face recognition; Image fusion; Match score fusion; Granular computing; Support vector machine; Dezert Smarandache theory

\section{Introduction}

Current face recognition systems capture faces of cooperative individuals in a controlled environment as part of the face recognition process. It is therefore possible to control the lighting, pose, background, and quality of images. Under these conditions, the performance of face recognition algorithms is greatly enhanced. However, there is still a need for more robust and efficient face recognition algorithms to address challenges such as changes in illumination, variations in pose and expression, variations in facial features due to aging, and altered appearances due to disguise [1].

Face recognition algorithms generally use visible spectrum images for recognition because they provide clear representation of facial features and face texture to differentiate between two individuals. However, visible spectrum images also possess several other properties which affect the performance of

\footnotetext{
* Corresponding author. Tel.: +1 3042930405.

E-mail addresses: richas@csee.wvu.edu (R. Singh), mayankv@csee.wvu.edu (M. Vatsa), noore@csee.wvu.edu, afzel.noore@mail.wvu.edu (A. Noore).
}

recognition algorithms. For example, changes in lighting affect the representation of visible spectrum images and can influence feature extraction. Other variations in face images such as facial hairs, wrinkles, and expression are also evident in visible spectrum images and these variations increase the false rejection rate of face recognition algorithms. To address the challenges posed by visible spectrum images, researchers have used infrared images for face recognition [2-5]. Among all the infrared spectrum images, long wave infrared (LWIR) images possess several properties that are complementary to visible images. LWIR or thermal images are captured in the range of $8-12 \mu \mathrm{m}$. These images represent the heat pattern of the object and are invariant to illumination and expression. Face images captured in LWIR spectrum have less intra-class variation and help to reduce the false rejection rate of recognition algorithms. These properties of LWIR and visible images can be combined to improve the performance of face recognition algorithms.

In literature, researchers have compared the performance of visible and thermal face recognition using several face recognition algorithms. These results show that for variation in expression and illumination, thermal images provide better recognition performance compared to visible images $[2,6,7]$. 
Further, several fusion algorithms have been proposed to fuse the information extracted from visible and LWIR face images at image level [8-11], feature level [10-12], match score level [12], and decision level [12]. Information fusion of multispectral images provides better performance compared to either visible or infrared spectrum images. However, research in multispectral information fusion is relatively new and intelligent techniques such as granular computing, support vector machine (SVM), and theory of evidence are not explored. These intelligent techniques can enhance the recognition performance by providing better generalization capabilities to handle imprecise information.

In this paper, we propose algorithms to fuse LWIR and visible face images at image level and match score level. We first propose the formulation of $2 v$-granular SVM (2v-GSVM) for pattern classification which is used in the proposed image fusion algorithm. The proposed image fusion algorithm learns the properties of the multispectral face images at different resolution and granularity levels to determine optimal information and combines them to generate a fused image. We then apply 2D log polar Gabor [13] and local binary pattern [14] face recognition algorithms to extract global and local features from the fused image. The match scores obtained by matching these features are fused using the proposed Dezert Smarandache (DSm) fusion algorithm [15,16]. The proposed DSm match score fusion algorithm is based on evidence theory which performs fusion depending on the evidence and belief of the match scores. On the Notre Dame [17,18] and Equinox [19] face databases, this integrated hierarchical scheme yields verification accuracy of more than $99.5 \%$.

We have organized the proposed algorithms in four sections. Section 2 describes the proposed formulation of $2 v$-GSVM. In Section 3, we describe the proposed visible and infrared face image fusion algorithm which dynamically and locally computes the weights using $2 v$-GSVM to generate the fused face image. We then describe the overview of DSm theory and the proposed DSm match score fusion algorithm in Section 4. Finally in Section 5, we combine the two levels of fusion to present the integrated multilevel image fusion and match score fusion algorithm. Section 6 describes the databases and existing algorithms used for validation of the proposed algorithms. The experimental results are summarized in Section 7.

\section{2v-granular support vector machine}

SVM is widely used in classification problems because it is designed to circumvent the overfitting problem [20-22] and is generalized to optimally perform classification on new training data. In literature, different variants of SVM have been proposed such as SVM, $v$-SVM, and dual $v$-SVM ( $2 v$-SVM). These variants are designed to improve the classification accuracy and address other challenges such as reduction in time complexity and classification with disparate number of training samples per class. In our previous research [12], we used $2 v$-SVM for feature fusion, match score fusion, and expert fusion. We observed that $2 v$-SVM provides better classification accuracy compared to classical SVM and is computationally more efficient. Recently, Tang et al. [23-26] applied the concept of granular computing [27-30] to SVM and proposed GSVM which is more adaptive to the data distribution in comparison to SVM. Tang et al. have also shown that for several classification applications, GSVM outperforms SVM both in terms of classification accuracy and computational time. In this paper, we extend the formulation to $2 v$-GSVM which embodies the properties of both GSVM and $2 v$-SVM. We first describe the formulation of $2 v$-SVM [22] followed by the granular modeling of $2 v$-SVM.

Let $\left\{\mathbf{x}_{i}, y_{i}\right\}$ be a set of $N$ data vectors with $\mathbf{x}_{i} \in \mathfrak{R}^{d}, y_{i} \in$ $(+1,-1)$, and $i=1, \ldots, N . \mathbf{x}_{i}$ is the $i$ th data vector that belongs to the binary class $y_{i}$. According to Chew et al. [22] the objective of training $2 v-\mathrm{SVM}$ is to find the hyperplane that separates two classes with the widest margins, i.e.,

$\mathbf{w} \varphi(\mathbf{x})+b=0$

subject to

$y_{i}\left(\mathbf{w} \varphi\left(\mathbf{x}_{i}\right)+b\right) \geqslant\left(\rho-\psi_{i}\right), \quad \rho, \psi_{i} \geqslant 0$

to minimize,

$\frac{1}{2}\|\mathbf{w}\|^{2}-\sum_{i} C_{i}\left(v \rho-\psi_{i}\right)$,

where $\rho$ is the position of the margin and $v$ is the error parameter. $\boldsymbol{\varphi}(\mathbf{x})$ is the mapping function used to map the data space to the feature space and provide generalization for the decision function that may not be a linear function of the training data. $C_{i}\left(v \rho-\psi_{i}\right)$ is the cost of errors, $\mathbf{w}$ is the normal vector, $b$ is the bias, and $\psi_{i}$ is the slack variable for classification errors. Slack variables are introduced to handle classes which cannot be separated by a hyperplane. Let $v_{+}$and $v_{-}$be the error parameters for training the positive and negative classes, respectively. Using these, the error parameter, $v$, is calculated as

$v=\frac{2 v_{+} v_{-}}{v_{+}+v_{-}}, \quad 0<v_{+}<1$ and $0<v_{-}<1$.

Error penalty $C_{i}$ is defined as

$C_{i}= \begin{cases}C_{+} & \text {if } y_{i}=+1, \\ C_{-} & \text {if } y_{i}=-1,\end{cases}$

where

$C_{+}=\left[n_{+}\left(1+\frac{v_{+}}{v_{-}}\right)\right]^{-1}$,

$C_{-}=\left[n_{-}\left(1+\frac{v_{-}}{v_{+}}\right)\right]^{-1}$

and $n_{+}$and $n_{-}$are the number of training points for the positive and negative classes, respectively. Further, the Wolfe dual formulation of $2 v-\mathrm{SVM}$ can be written as

$L=\sum_{i} \alpha_{i}-\left[\frac{1}{2} \sum_{i, j} \alpha_{i} \alpha_{j} y_{i} y_{j} K\left(x_{i}, x_{j}\right)\right]$,

where $i, j \in 1, \ldots, N, \alpha_{i}, \alpha_{j}$ are the Lagrange multipliers and $K(\cdot)$ is the kernel function. Finally, iterative decomposition 
training based optimization algorithm [22] is used to train the $2 v$-GSVM.

In this paper, we extend the formulation of $2 v-\mathrm{SVM}$ by using the granular computing approach similar to Ref. [25]. Granular computing is a knowledge-oriented divide and conquer approach to problem solving Refs. [27-30]. In granular computing, information is divided into subproblems called granules and these subproblems are solved individually at different granularity levels. Using this concept, $2 v$-GSVM is formulated as follows.

Let the complete feature space be divided into $k$ subspaces with one $2 v$-SVM operating on each subspace. The $i$ th $2 v$-SVM is represented by $2 v \mathrm{SVM}_{i}$, where $i=1,2, \ldots, k$. From each of the subspace, we obtain the corresponding $L_{i}$ using Eq. (8). We then compute the compound margin width $W$ by using all the $L_{i}$ values:

$W=\left|\sum_{i=1}^{k} \frac{t_{i}}{t}\left(2 v \mathrm{SVM}_{i}: \rightarrow L_{i}\right)-L_{0}\right|$,

where $t_{i}$ is the number of training data in the $i$ th subspace and $t=\sum_{i=1}^{k} t_{i} .2 v \mathrm{SVM}_{i}: \rightarrow L_{i}$ represents the SVM operating on the $i$ th subspace. $2 v$-SVM learning yields $L_{i}$ at local level, and $L_{0}$ is obtained by learning another $2 v$-SVM on the complete feature space at global level. This equation provides the margin width associated to a hyperplane. There are different methods to divide the feature space and hence different hyperplanes associated with each of the granule generation method can be obtained. We compute the classification accuracy of all the hyperplanes on the training data and then select the hyperplane that optimally classifies the training data. In contrast to a single SVM that deals with large parameter space and results in large training time, $2 v$-GSVM uses multiple SVMs to learn both the local and global properties of the training data at different granularity levels. This $2 v$-GSVM is then used for fusing multispectral face images.

\section{Multispectral face image fusion using $2 v$-GSVM}

In multispectral face recognition, visible images provide the reflectance property and LWIR images provide the thermal property. In $2 v$-GSVM based image fusion, we combine these properties to generate a fused image which possess both the properties and can be used to improve the recognition performance. Although there are several multispectral face image fusion algorithms in literature, they have some limitations which affect the face recognition performance. Genetic algorithm based fusion proposed by Bebis et al. [31] suffers from making a good choice of fitness function. Fusion algorithm proposed by Kong et al. [4] suffers from the empirical constant weights which are assigned to the wavelet coefficients of visible and LWIR images. In real world applications, weights should be dynamically and locally assigned for optimal multispectral information fusion. In this section, we propose the multispectral face image fusion algorithm which dynamically and locally computes the weights for fusion using $2 v$-GSVM. Fig. 1 illustrates the steps involved in the proposed image fusion algorithm. The algorithm is divided into two steps: image registration and image fusion.

\subsection{Mutual information based multispectral face image fusion}

Visible and infrared images captured at different time instances can have variations due to camera angle, expression, and geometric deformations. To optimally fuse two multispectral images, we first need to minimize the linear and non-linear differences between the two images. In this section, we propose the use of mutual information based registration algorithm for registering visible and thermal face images. Mutual information is a concept from information theory in which statistical dependence is measured between two random variables. Researchers in medical imaging have used mutual information based registration algorithms to effectively fuse images from different modalities such as CT and MRI [32,33]. Registration of multispectral face images is described as follows.

Let $V$ and $I$ be the input visible and infrared face images for registration. Mutual information between the two face images can be represented as

$M(V, I)=H(V)+H(I)-H(V, I)$,

$H(\cdot)$ is the entropy of the image and $H(V, I)$ is the joint entropy. Registering $V$ with respect to $I$ requires maximization of mutual information between $I$ and $V$, thus maximizing the entropy $H(V)$ and $H(I)$, and minimizing the joint entropy $H(V, I)$. Mutual information based registration algorithms are sensitive to changes that occur in the distributions as a result of difference in overlap regions. To address this issue, Studholme et al. [34] proposed normalized mutual information which can be represented as

$N M(V, I)=\frac{H(V)+H(I)}{H(V, I)}$.

The registration is performed on a transformation space, $T$, such that

$T=\left[\begin{array}{lll}a & b & 0 \\ c & d & 0 \\ e & f & 1\end{array}\right]$,

where $a, b, c, d$ are the shear, scale, and rotation parameters, and $e, f$ are the translation parameters. Using the normalized mutual information, we define a search strategy to find the transformation parameters, $T^{*}$, by exploring the search space, $T$ :

$T^{*}=\arg \max _{\{T\}}\{N M(I, T(V))\}$.

Multispectral face images $V$ and $I$ are thus registered using the transformation parameters $T^{*}$. This registration algorithm is linear in nature. To accommodate the non-linear variation in face images, we apply multiresolution image pyramid scheme in which we first build Gaussian pyramid of both visible and thermal face images. Registration parameters are estimated at the coarsest level and used to warp the face image in the next level of the pyramid. The process is iteratively repeated through each level of the pyramid and a final transformed visible face 


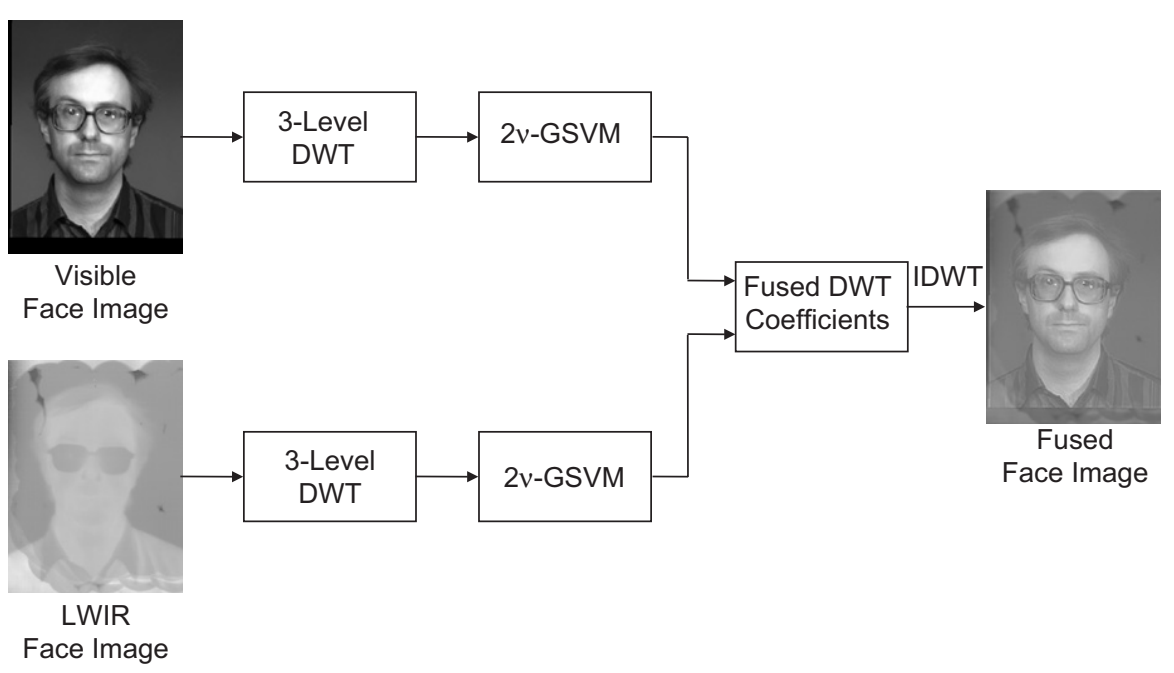

Fig. 1. Schematic diagram of the proposed $2 v$-GSVM image fusion algorithm.

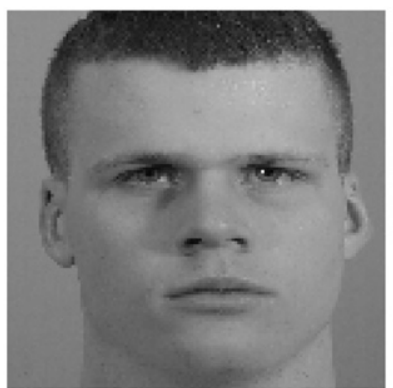

Visible

Face Image

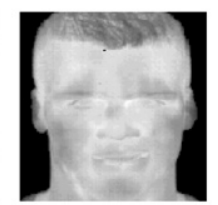

LWIR

Face Image

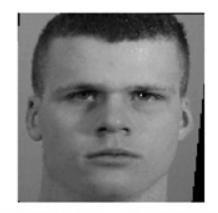

Registered Visible Face Image
Fig. 2. Example of visible and infrared image registration on the Notre Dame face database [18]. Visible image is registered with respect to the LWIR image. Size of detected visible face image is $855 \times 1024$ and infrared face image is $115 \times 156$.

image is obtained at the finest pyramid level. In this manner, we handle the large global variations at the coarsest resolution level and local non-linear variations at the finest resolution level. Fig. 2 shows examples of the registration algorithm in which visible face image is registered with respect to LWIR face image.

\subsection{Proposed 2v-GSVM image fusion algorithm}

In the proposed face image fusion algorithm, registered visible and infrared face images are fused using $2 v$-GSVM and discrete wavelet transform (DWT) [35]. The fusion algorithm uses the activity level, $a$, of face images which is defined as where $V$ is the visible face image, and $X$ and $Y$ are the rows and columns of the face image, respectively. The proposed fusion algorithm is divided into two parts: (1) training and (2) classification and fusion.

Training 2v-GSVM: We learn the 2v-GSVM for image fusion by using the activity levels of labeled visible and infrared training face images. The training algorithm is described as follows:

Step 1: Visible and infrared training face images are decomposed using DWT to obtain three-level approximation, horizontal, vertical, and diagonal subbands.

Step 2: Let $V_{L L_{j}}, V_{L H_{j}}, V_{H L_{j}}$, and $V_{H H_{j}}$ be the subbands of visible face image where $j=1,2,3$ represents the decomposition levels. Similarly, let $I_{L L_{j}}, I_{L H_{j}}, I_{H L_{j}}$, and $I_{H H_{j}}$ be the subbands of infrared face image corresponding to each decomposition level, $j$. Each subband of both visible and infrared face images is divided into windows of size $8 \times 8$ and the activity level of each window is computed using Eq. (14).

Step 3: The activity levels of all labeled training face images are used as input to $2 v$-GSVM. In training, two $2 v$-GSVMs are learned, one for visible face images and another for infrared face images.

Step 4: $2 v$-GSVM trained for visible images classifies the activity levels of visible spectrum face images as Good or +1 and $\mathrm{Bad}$ or -1 . Similarly, $2 v$-GSVM trained for infrared face images classifies the activity levels of infrared face images into Good or Bad class.

Classification and fusion: We classify the properties of visible and infrared face images using trained $2 v$-GSVMs. This classification is used to dynamically compute the weights of visible and infrared face images in multispectral image fusion.

Step 1: Visible and infrared face images of an individual are provided as input. Similar to Steps 1 and 2 of the training algorithm, both the input face images are decomposed into

$a=\sqrt{\frac{1}{X Y}\left[\sum_{i=0}^{X-1} \sum_{j=1}^{Y-1}\left\{(V(i, j)-V(i, j-1)\}^{2}+\sum_{j=0}^{Y-1} \sum_{i=1}^{X-1}\left\{(V(i, j)-V(i-1, j)\}^{2}\right]\right.\right.}$, 
three-level DWT and activity levels of $8 \times 8$ windows are computed. Let $a_{V}$ and $a_{I}$ be the activity levels computed from visible and infrared face images, respectively.

Step 2: $2 v$-GSVM classifier is used to classify the activity levels of different subbands of visible face images as Good or $\mathrm{Bad}$. A binary decision matrix, $d_{V}$, is generated which contains value 1 if the activity level is Good and 0 if the activity level is $\mathrm{Bad}$.

Step 3: Similar to Step 2, activity levels of infrared face image are classified and a binary decision matrix, $d_{I}$, is generated.

Step 4: Weight matrices $\omega_{V}$ and $\omega_{I}$ are computed using binary decision matrices $\left(d_{V}\right.$ and $\left.d_{I}\right)$ and the following three conditions:

(1) If $d_{V}(i)=d_{I}(i)=1$, then $\omega_{V}(i)=\omega_{I}(i)=0.5$.

(2) If $d_{V}(i)=1$ and $d_{I}(i)=0$, then $\omega_{V}(i)>\omega_{I}(i)$ and

$$
\begin{aligned}
& \omega_{V}(i)=\frac{\left|a_{V}(i)+2 a_{I}(i)-a_{I_{\text {median }}}\right|}{a_{V}(i)+a_{I}(i)}, \\
& \omega_{I}(i)=\frac{\left|a_{I_{\text {median }}}-a_{I}(i)\right|}{a_{V}(i)+a_{I}(i)} .
\end{aligned}
$$

(3) If $d_{V}(i)=0$ and $d_{I}(i)=1$, then $\omega_{V}(i)<\omega_{I}(i)$ and

$$
\begin{aligned}
& \omega_{V}(i)=\frac{\left|a_{V_{\text {median }}}-a_{V}(i)\right|}{a_{V}(i)+a_{I}(i)}, \\
& \omega_{I}(i)=\frac{\left|a_{I}(i)+2 a_{V}(i)-a_{V_{\text {median }}}\right|}{a_{V}(i)+a_{I}(i)},
\end{aligned}
$$

where $i$ is the window count, and $a_{V_{\text {median }}}$ and $a_{I_{\text {median }}}$ are the median values of $a_{V}$ and $a_{I}$ matrices, respectively. Further, in all three cases, $\omega_{V}(i)+\omega_{i}(i)=1$.

In condition 1, the activity levels of both visible and infrared image windows are classified as Good and hence equal weights are assigned. Condition 2 states that if the activity level of window corresponding to visible face image is classified as Good and the activity level of the window corresponding to infrared face image is classified as Bad, then higher weight is assigned to the visible face image window. In condition 3, higher weight is assigned to the infrared face image window because $2 v$-GSVM classifies the activity level of visible face image window as Bad and the activity level of infrared face image window as Good.

Step 5: Visible and infrared face images are then fused using

$F_{j 8 \times 8}(i)=\omega_{V}(i) V_{j 8 \times 8}(i)+\omega_{I}(i) I_{j 8 \times 8}(i)$,

where $F_{j}$ is the fused subband, $j$ represents the approximate, vertical, horizontal, and diagonal subbands, subscript $8 \times 8$ denotes that the fusion is performed at window level of size $8 \times 8$, and $i$ represents the window count.

Step 6: Finally, inverse DWT is applied on fused subbands to generate the fused multispectral face image, F. Fig. 3 shows an example of visible, infrared, and fused face images of an individual.

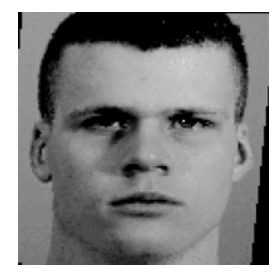

Registered Visible Face Image
LWIR Face Image

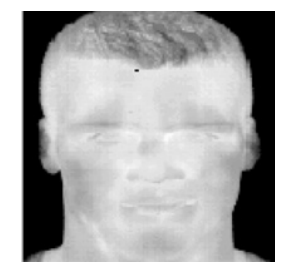

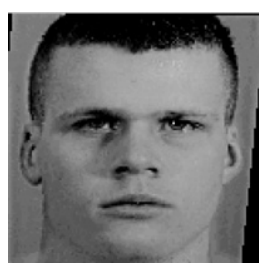

Fused Face Image
Fig. 3. Sample result of the proposed image fusion algorithm.

\section{Match score fusion using DSm theory}

In multimodal biometrics, researchers have proposed several match score fusion algorithms such as AND/OR rule [36], Sum rule [36], and SVM fusion [37]. Another mathematical paradigm of information fusion is based on the theory of evidence. Dempster Shafer theory (DST) [38] based fusion algorithm is one example of this paradigm in which uncertain and fuzzy information are efficiently fused. In multimodal biometrics, it has been shown that DST based fusion algorithms perform better compared to existing fusion algorithms [39]. However, DST has some limitations as reported by Zadeh [40-42], Dubois and Prade [43], and Voorbraak [44]. Researchers have shown that results for DST are not trustworthy when conflict between different sources is large. Other limitations related to Dempster rule of combination are reported in Ref. [15]. Recently, DSm theory based fusion algorithm $[15,16]$ has been proposed to circumvent the limitations of other evidence theory based fusion algorithms. DSm theory is a powerful mathematical model for information fusion which includes both Bayes theory and DST as special cases. In this section, we first present a brief overview of DSm theory and hybrid DSm rule of combination [16] followed by the proposed match score fusion algorithm.

\subsection{Overview of DSm theory}

DSm theory is a powerful tool for representing and fusing uncertain or conflicting knowledge. It can solve complex static or dynamic fusion problems using plausible and paradoxical reasoning $[15,16]$. Since identity verification is a two class problem with the classes being genuine and impostor, we explain DSm theory for a two class problem.

Let $\Theta=\left\{\theta_{1}, \theta_{2}\right\}$ be the frame of discernment which consists of a finite set of exhaustive and mutually exclusive hypothesis. Hyperpower set of the frame of discernment is defined as $D^{\Theta}=$ $\left\{\varnothing, \theta_{1}, \theta_{2}, \theta_{1} \cup \theta_{2}, \theta_{1} \cap \theta_{2}\right\}$. A mapping $m(\cdot)$ on $\Theta$ is defined as $m(\cdot)=D^{\Theta} \rightarrow[0,1]$, such that

$$
\sum_{A \in D^{\Theta}} m(A)=1,
$$

$m(A)$ is called the generalized basic belief assignment (gbba) of $A$ and $m(\emptyset)=0$. Further, a generalized belief function $\mathrm{Bel}$ 
is a mapping function $\mathrm{Bel}: D^{\Theta} \rightarrow[0,1]$ such that

$\operatorname{Bel}(A)=\sum_{X \subseteq A, X \in D^{\Theta}} m(X)$.

More specifically,

$\operatorname{Bel}(A)_{y, t}^{\Theta, D^{\Theta}}\left[E_{y, t}\right]\left(w_{0} \in A\right)=x$.

This equation denotes the degree of belief $x$ of the classifier $y$ at time $t$ when $w_{0}$ belongs to $A$ and $A \in D^{\Theta}$. Belief is based on evidential corpus $E_{y, t}$ held by $y$ at time $t$. To simplify, generalized belief function can also be written as $\operatorname{Bel}(A)$. Further, generalized belief function, $\mathrm{Bel}$, uniquely corresponds to gbba $m$ and vice versa.

For fusing two information sources, $X$ and $Y$, the DSm rule of combination [16] is defined as

$m_{M(\Theta)}(A)=\psi(A)\left[S_{1}(A)+S_{2}(A)+S_{3}(A)\right]$,

where $M(\Theta)$ is the model over which DSm theory operates and $\psi(A)$ is the characteristic non-emptiness function of $A$ which is 1 if $A \notin \emptyset$ and 0 otherwise. $S_{1}(A), S_{2}(A)$, and $S_{3}(A)$ are defined as

$$
\begin{aligned}
& S_{1}(A)=\sum_{\left(X, Y \in D^{\Theta}, X \cap Y=A\right)} m_{1}(X) m_{2}(Y), \\
& S_{2}(A)=\sum_{\left(X, Y \in \boldsymbol{\Phi},[v=A] \vee\left[(v \in \boldsymbol{\Phi}) \wedge\left(A=I_{t}\right)\right]\right)} m_{1}(X) m_{2}(Y), \\
& S_{3}(A)=\sum_{\left(X, Y \in D^{\Theta}, X \cup Y=A, X \cap Y \in \boldsymbol{\Phi}\right)} m_{1}(X) m_{2}(Y),
\end{aligned}
$$

where $I_{t}$ is total ignorance and is the union of all $\theta_{i}(i=1,2)$, i.e., $I_{t}=\theta_{1} \cup \theta_{2} . \boldsymbol{\Phi}=\{\boldsymbol{\Phi}, \phi\}$ is the set of all elements of $D^{\Theta}$ which are empty under the constraints of some specific problem, and $\phi$ is the empty set. $v=u(X) \cup u(Y)$, where $u(X)$ is the union of all singletons $\theta_{i}$ that compose $X$ and $Y$. Here, $S_{1}(A)$ corresponds to the classical DSm rule on the free DSm model [15], $S_{2}(A)$ represents the mass of all relatively and absolutely empty sets which is transferred to the total or relative ignorance, and $S_{3}(A)$ transfers the sum of relative empty sets to the non-empty sets. An excellent description of DSm theory is presented in Ref. [16].

Probability based approaches have limitations because they deal with basic probability assignment $m(\cdot) \in[0,1]$ and $m\left(\theta_{1}\right)+m\left(\theta_{2}\right)=1$. Further, DST deals with basic belief assignment $m(\cdot) \in[0,1]$ such that $m\left(\theta_{1}\right)+m\left(\theta_{2}\right)+m\left(\theta_{1} \cup \theta_{2}\right)=1$. In contrast, DSm theory is more generalized and deals with belief functions associated with the generalized belief assignment such that $m\left(\theta_{1}\right)+m\left(\theta_{2}\right)+m\left(\theta_{1} \cup \theta_{2}\right)+m\left(\theta_{1} \cap \theta_{2}\right)=1$.

\subsection{Proposed multimodal match score fusion algorithm}

In this section, we propose a novel match score fusion algorithm using DSm theory. Fig. 4 shows the steps involved in the proposed match score fusion algorithm for a single image. Two match scores are computed by matching the global features and local features extracted from probe and gallery face images. These two match scores are fused using DSm theory. We first define:

- Frame of discernment: $\Theta=\left\{\theta_{\text {genuine }}, \theta_{\text {impostor }}\right\}$.

- Dedekind lattice: $D^{\Theta}=\left\{\theta_{\text {genuine }}, \theta_{\text {impostor }}, \theta_{\text {genuine }} \cup\right.$ $\left.\theta_{\text {impostor }}, \theta_{\text {genuine }} \cap \theta_{\text {impostor }}\right\}$.

Let $s_{1}$ and $s_{2}$ be the two match scores computed from two face recognition algorithms. Let us assume that the distribution of match scores to an element of $D^{\Theta}$ is a Gaussian distribution

$p\left(s_{i}, \mu_{i j}, \sigma_{i j}\right)=\frac{1}{\sigma_{i j} \sqrt{2 \pi}} \exp \left[-\frac{1}{2}\left\{\frac{s_{i}-\mu_{i j}}{\sigma_{i j}}\right\}^{2}\right]$,

where $\mu_{i j}$ and $\sigma_{i j}$ are the mean and standard deviation of the $i$ th classifier corresponding to the $j$ th element of $D^{\Theta}$. We use this Gaussian distribution to compute the gbba,

$m_{i}(\cdot)=D^{\Theta} \backslash\left\{\theta_{\text {genuine }} \cup \theta_{\text {impostor }}\right\} \rightarrow[0,1]$.

Since in biometrics, a match score can only belong to genuine $\left(\theta_{\text {genuine }}\right)$, impostor $\left(\theta_{\text {impostor }}\right)$, or conflicting region $\left(\theta_{\text {genuine }} \cap\right.$ $\left.\theta_{\text {impostor }}\right)$, we set $m_{i}\left(\theta_{\text {genuine }} \cup \theta_{\text {impostor }}\right)=0.001$ and compute the remaining gbbas as follows:

$m_{i}(j)=\frac{p\left(s_{i}, \mu_{i j}, \sigma_{i j}\right) \beta_{i j}}{\sum_{j=1}^{D^{\Theta} \mid-1} p\left(s_{i}, \mu_{i j}, \sigma_{i j}\right) \beta_{i j}}$,

where $\beta_{i j}$ is the prior of classifier $i$ corresponding to the $j$ th element of $D^{\Theta} \backslash\left\{\theta_{\text {genuine }} \cup \theta_{\text {impostor }}\right\}$. We have used $\beta_{i j}$ as the verification accuracy computed on the training database and its value lies in the range of $(0,1)$. Gbbas of the two classifiers $m_{1}(\cdot)$ and $m_{2}(\cdot)$ are computed and fused using

$m_{\text {fused }}=m_{1} \oplus m_{2}$,

where $\oplus$ represents the hybrid DSm rule of combination defined in Eq. (23), Section 4.1. Finally, threshold $T$ is used to classify the decision as accept or reject:

Decision $= \begin{cases}\text { accept } & \text { if } m_{\text {fused }} \geqslant T, \\ \text { reject } & \text { otherwise. }\end{cases}$

To extend this algorithm to multispectral face images, we separately apply the feature extraction algorithms to the visible face image and the infrared face image. As shown in Fig. 5, we extract the global facial features using 2D log polar Gabor transform [13] and the local facial features using local binary pattern [14], and then match these features to compute the corresponding match scores. The two match scores for the visible face image are fused using the proposed DSm fusion algorithm. Similarly, the two match scores for the infrared face image are also fused to generate the fused match score. Finally, the fused match scores of the visible face image and the infrared face image are combined to compute a composite match score for multispectral face images. A decision of accept or reject is made using the composite match score. 


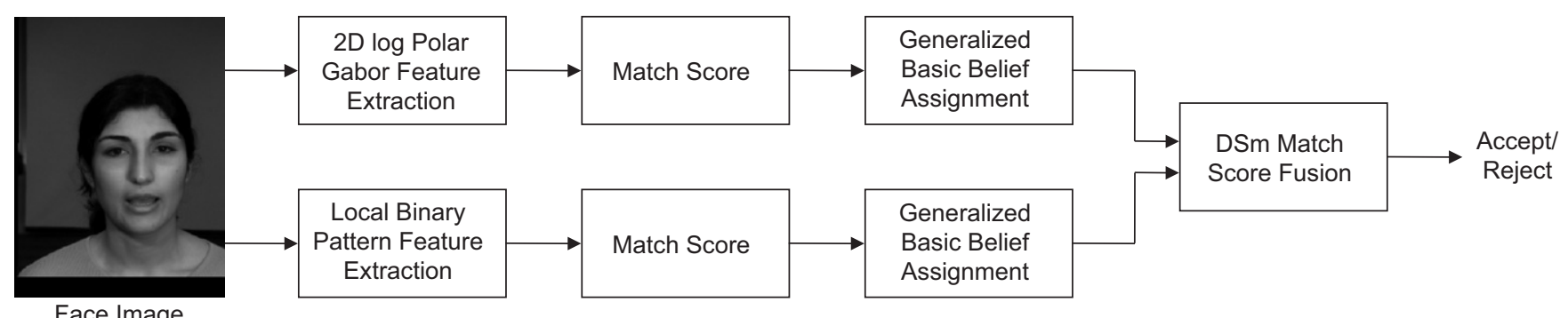

Face Image

Fig. 4. Block diagram of the proposed DSm match score fusion algorithm in which match scores obtained from two classifiers are fused.
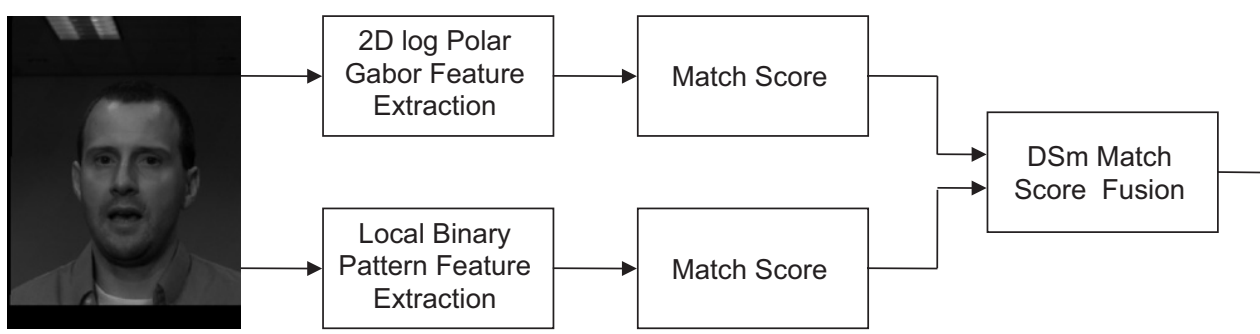

Visible

Face Image
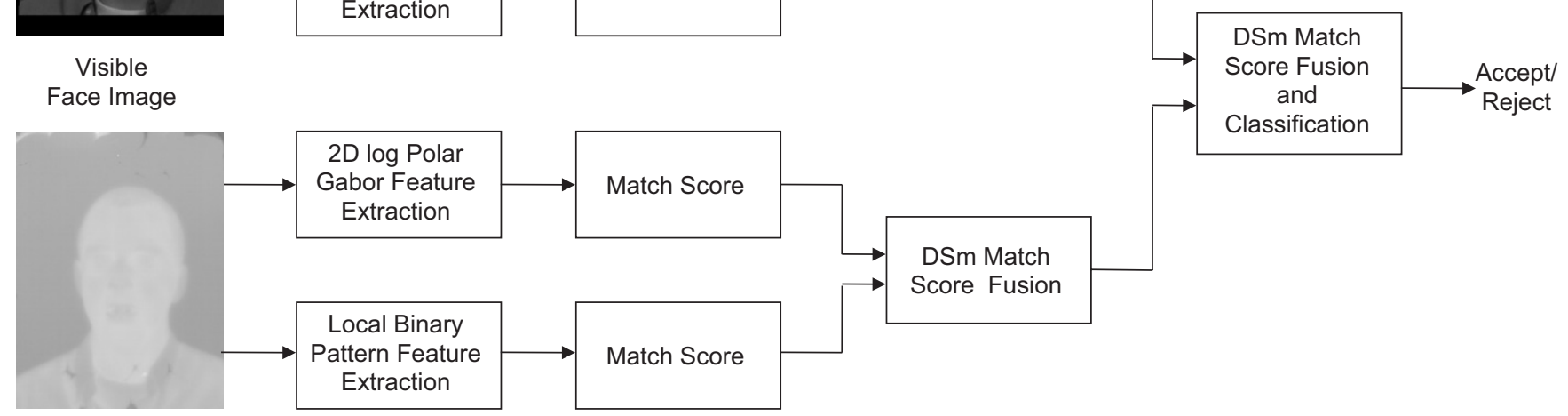

LWIR

Face Image

Fig. 5. Steps involved in the proposed DSm match score fusion algorithm. In this case, match scores obtained by applying the two classifiers on multispectral face images are fused.

\section{Integration of image fusion and match score fusion}

The proposed image and match score level fusion algorithms are integrated to further improve the verification performance. Fig. 6 shows the block diagram of the integrated multilevel fusion algorithm. Visible and infrared face images are first fused using the proposed $2 v$-GSVM image fusion algorithm described in Section 3. 2D log polar Gabor transform [13] and local binary pattern [14] feature extraction algorithms are then applied on the fused multispectral face image to extract global and local facial features. Match scores computed by matching these features are fused using the proposed DSm match score fusion algorithm described in Section 4.

\section{Databases and existing algorithms used for validation}

To validate the proposed fusion algorithms, we used the Notre Dame [17,18] and Equinox/NIST [19] multispectral face databases. In addition, we used 2D log polar Gabor transform [13] and local binary pattern algorithms [14] for face verification. For comparing the performance of the proposed algorithms, we used several existing image fusion and match score fusion algorithms. In this section, we briefly describe the details of the databases and algorithms used in our experiments.

\subsection{Databases used for validation}

- Notre Dame face database: Notre Dame face database $[17,18]$ contains LWIR and visible images from 159 classes with variations in expression, lighting, and time lapse. We have chosen three visible and LWIR face image with neutral expression for training database, one neutral visible and LWIR image for gallery database, and the remaining images comprise the probe data set. Table 1 shows the details of training, gallery, and probe images used in the experiments.

- Equinox face database: Equinox face database [19] contains LWIR, medium wave infrared, short wave infrared, and visible face images pertaining to 95 individuals. LWIR images are captured at $8-12 \mu \mathrm{m}$, medium wave infrared images at 3-5 $\mu \mathrm{m}$, and short wave infrared images at $0.9-1.7 \mu \mathrm{m}$. The 


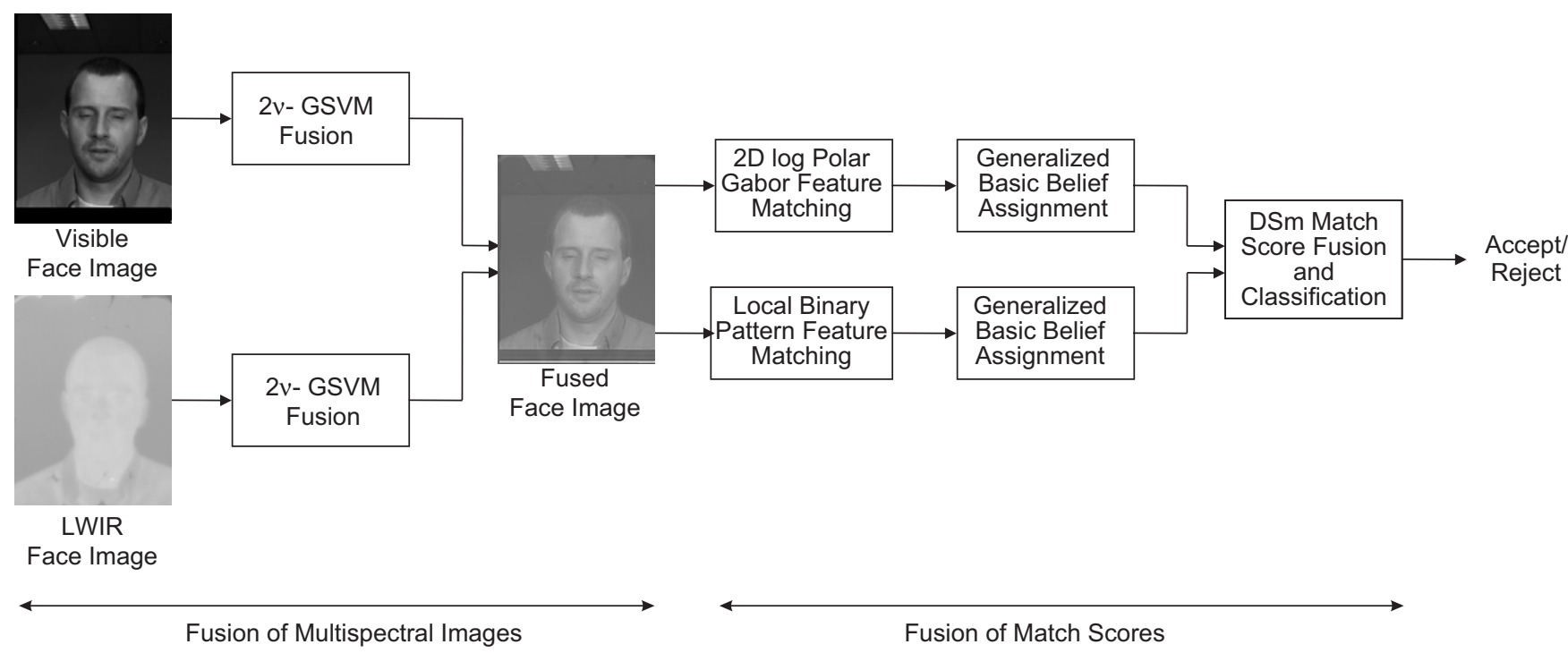

Fig. 6. Steps involved in the proposed multilevel image fusion and match score fusion algorithm.

Table 1

Number of visible and infrared image pairs in the training, gallery, and probe databases

\begin{tabular}{llcc}
\hline Face database & \multicolumn{2}{l}{ Number of visible and infrared image pairs in } \\
\cline { 2 - 4 } & Training database & Gallery database & Probe database \\
\hline Notre Dame & 477 & 159 & 1815 \\
Equinox & 285 & 95 & 18715 \\
\hline
\end{tabular}

images are captured under different illumination conditions and contain variations in expression and glasses. In our research, we have used only LWIR images and visible images. The number of LWIR and visible images per class vary from 43 to 516 . We chose three visible and LWIR images with neutral expression and without glasses for training, one LWIR and visible image with neutral expression, uniform illumination, and no glasses for gallery, and the remaining images as probe.

\subsection{Face recognition algorithms}

We first detect the face region from input images. Visible face images are detected using the triangle based face detection algorithm [45] whereas a thresholding based face detection algorithm [4] is applied to detect LWIR face images. Global and local facial features are extracted from these detected face images using the face recognition algorithms described below.

- 2D log polar Gabor transform: In the 2D log polar Gabor transform based face recognition algorithm, the face image is transformed into polar coordinates and textural features are extracted using the 2D log polar Gabor transform [13]. These features are matched using the Hamming distance to generate match scores.

- Local binary pattern: In this algorithm, a face image is divided into several regions and weighted LBP features are extracted to generate a feature vector [14]. Matching of two LBP feature vectors is performed using weighted Chi square distance measure algorithm.

\subsection{Existing fusion algorithms}

To compare the performance of the proposed $2 v$-GSVM based image fusion algorithm, we chose image fusion algorithms proposed by Kong et al. [4] and Singh et al. [10]. Both the algorithms use DWT to fuse infrared and visible face images. To compare the performance of the proposed DSm based match score fusion algorithm, we chose four existing fusion algorithms, Product rule [36], Sum rule [36], SVM fusion [37], and DST fusion [39]. Product rule and Sum rule are based on statistical rules, SVM fusion algorithm is a learning based algorithm, and DST fusion algorithm is based on evidence theory.

\section{Experimental validation of the proposed algorithms}

In this section, we perform the experiments to validate the proposed image fusion and match score fusion algorithms. Using the training images, we train both $2 v$-GSVM image fusion algorithm and DSm match score fusion algorithm. For $2 v$-GSVM learning and classification, we used the radial basis function $(\mathrm{RBF})$ kernel with RBF parameter as 4 . The performance is evaluated in terms of verification accuracy at $0.01 \%$ false accept rate (FAR). The experimental validation is divided into four parts:

(1) Validation of the proposed $2 v$-GSVM image fusion algorithm.

(2) Validation of the proposed DSm match score fusion algorithm.

(3) Validation of the integrated multilevel image fusion and match score fusion algorithm. 
Table 2

Verification performance of the proposed $2 v$-GSVM and existing image fusion algorithms at $0.01 \%$ FAR

\begin{tabular}{|c|c|c|c|c|c|c|}
\hline \multirow[t]{2}{*}{ Face database } & \multirow[t]{2}{*}{ Recognition algorithm } & \multicolumn{5}{|c|}{ Verification accuracy (\%) } \\
\hline & & Visible image & LWIR image & Kong image fusion [4] & Singh image fusion [10] & Proposed image fusion \\
\hline \multirow[t]{2}{*}{ Notre Dame } & 2D log polar Gabor & 89.36 & 88.09 & 86.74 & 91.88 & 95.85 \\
\hline & Local binary pattern & 88.20 & 87.44 & 85.87 & 91.79 & 94.80 \\
\hline \multirow[t]{2}{*}{ Equinox } & 2D log polar Gabor & 78.91 & 82.75 & 80.83 & 90.06 & 94.98 \\
\hline & Local binary pattern & 76.80 & 81.52 & 80.69 & 89.93 & 94.71 \\
\hline
\end{tabular}

(4) Statistical evaluation of the proposed fusion algorithms using half total error rate (HTER).

\subsection{Validation of the proposed $2 v-G S V M$ image fusion algorithm}

The performance of the proposed image fusion algorithm is evaluated using the Notre Dame and Equinox face databases. We compared the performance with two existing multispectral face image fusion algorithms referred to as Kong image fusion [4] and Singh image fusion [10]. For evaluation, we separately computed the verification accuracies of visible face image and LWIR face image using both 2D log polar Gabor and local binary pattern face verification algorithms. The third and fourth columns of Table 2 summarize the verification performance of visible face image and LWIR face image, respectively, using both the verification algorithms. These results establish the baseline for evaluating and comparing the performance of fusion algorithms. We then compute the verification accuracies with the proposed $2 v$-GSVM multispectral face image fusion algorithm and existing image fusion algorithms. The results summarized in Table 2 show that the proposed image fusion algorithm outperforms both the existing fusion algorithms by at least $3.9 \%$ for the Notre Dame database and $4.9 \%$ for the Equinox database. ROC plots in Figs. 7 and 8 show the results for the Notre Dame and Equinox face databases, respectively.

The proposed $2 v$-GSVM image fusion algorithm performs correct classification of multispectral face information at different levels of granularity which is subsequently used for computing the dynamic weights of visible and LWIR face images. This granular learning results in better generalization and fusion of high entropy visible and LWIR face features. Further, as shown in Fig. 9, fused face images generated from the proposed image fusion algorithm provide more invariance to illumination compared to the visible images. The fused images also provide more distinguishing information compared to the LWIR face images. These properties of the proposed $2 v$-GSVM image fusion algorithm lead to improved face verification performance.

\subsection{Validation of the proposed DSm match score fusion algorithm}

To validate the performance of the proposed DSm match score fusion algorithm, we compute the composite match score a

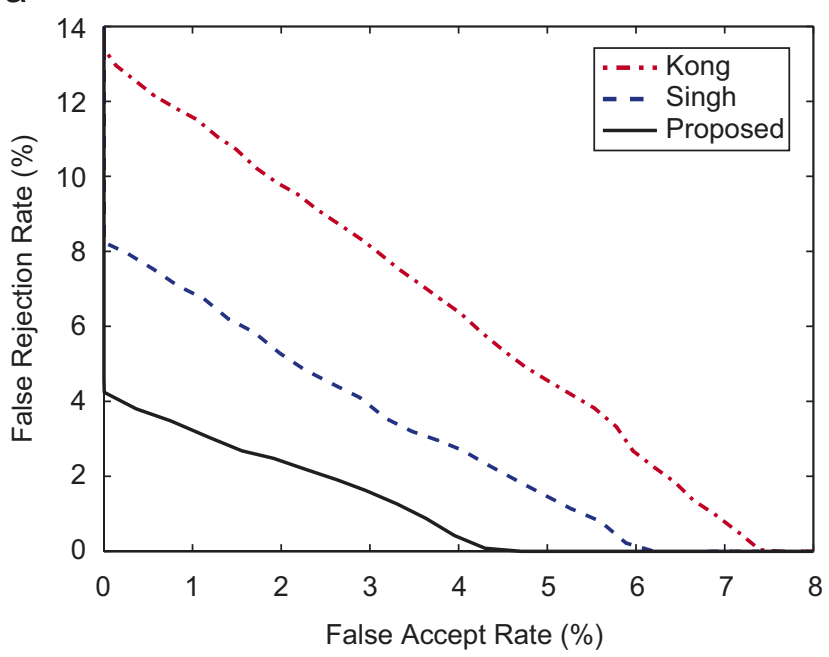

b

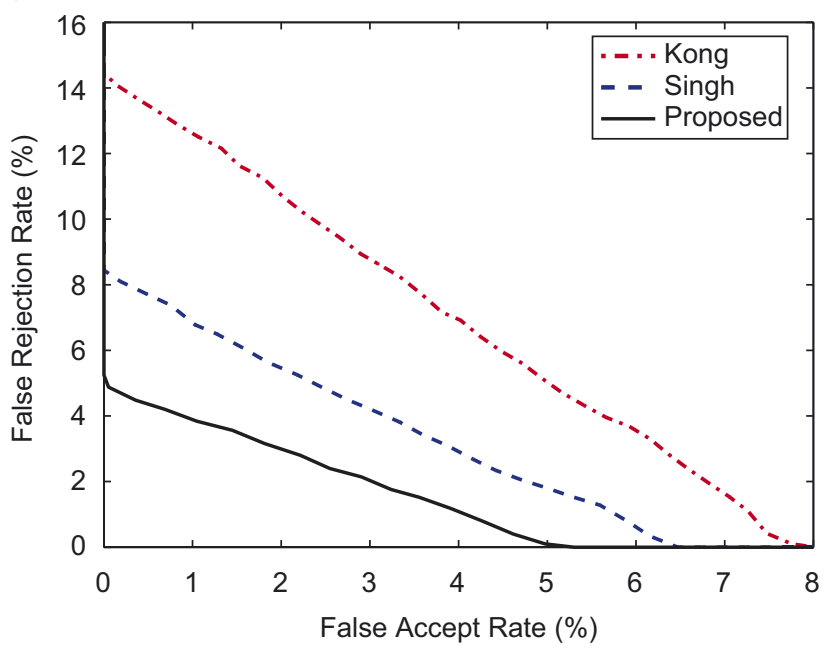

Fig. 7. ROC plots of the proposed $2 v$-GSVM and existing image fusion algorithms on the Notre Dame face database. Results are computed using (a) 2D log polar Gabor (b) local binary pattern based verification algorithms.

as described in Section 4. First the match score is obtained from the visible and the LWIR face images. Note that these images are not fused. However, as shown in Fig. 5, the fusion occurs at match score level. Next, the fused match scores obtained from each image are combined using the proposed DSm algorithm to generate the composite match score. The verification accuracies are summarized in Table 3 and Fig. 10. On both the databases, the proposed DSm match score fusion algorithm yields more than $98 \%$ accuracy. On the Notre Dame face 
a

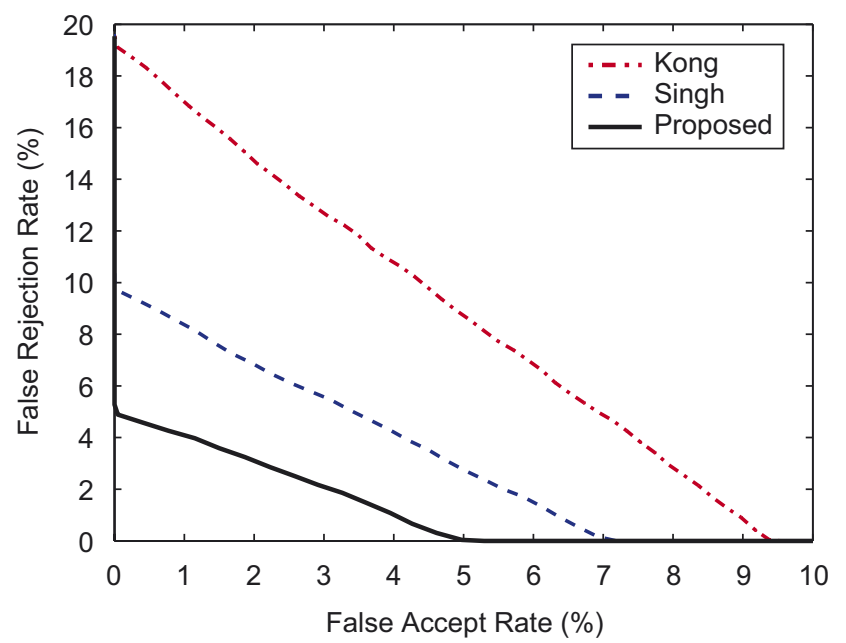

b

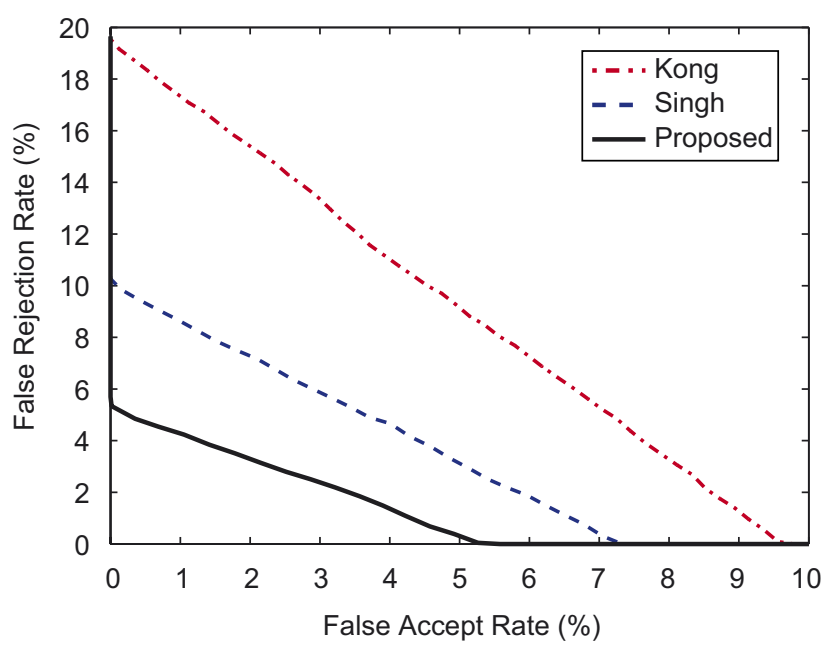

Fig. 8. ROC plots of the proposed 2v-GSVM and existing image fusion algorithms on the Equinox face database. Results are computed using (a) 2D log polar Gabor (b) local binary pattern based verification algorithms.

database, the proposed algorithm provides at least $1.24 \%$ better performance compared to the second best DST based match score fusion algorithm [39]. On the Equinox database, the proposed algorithm provides $1.57 \%$ and $3.03 \%$ better verification performance compared to DST and SVM fusion algorithms, respectively.

The performance of existing match score fusion algorithms decreases when two face recognition classifiers yield conflicting decisions. For example the classifier using global features may make a decision to accept and the classifier using local features may make a decision to reject. In such cases, the proposed DSm match score fusion algorithm operates on the intersection region ( $\left.\theta_{\text {genuine }} \cap \theta_{\text {impostor }}\right)$ to make an optimal decision using the prior information of classifiers. Existing statistical and learning based fusion algorithms including DST fusion algorithm do not account for the conflicting region. Hence, the plausible and paradoxical reasoning technique of DSm theory provides better verification performance.
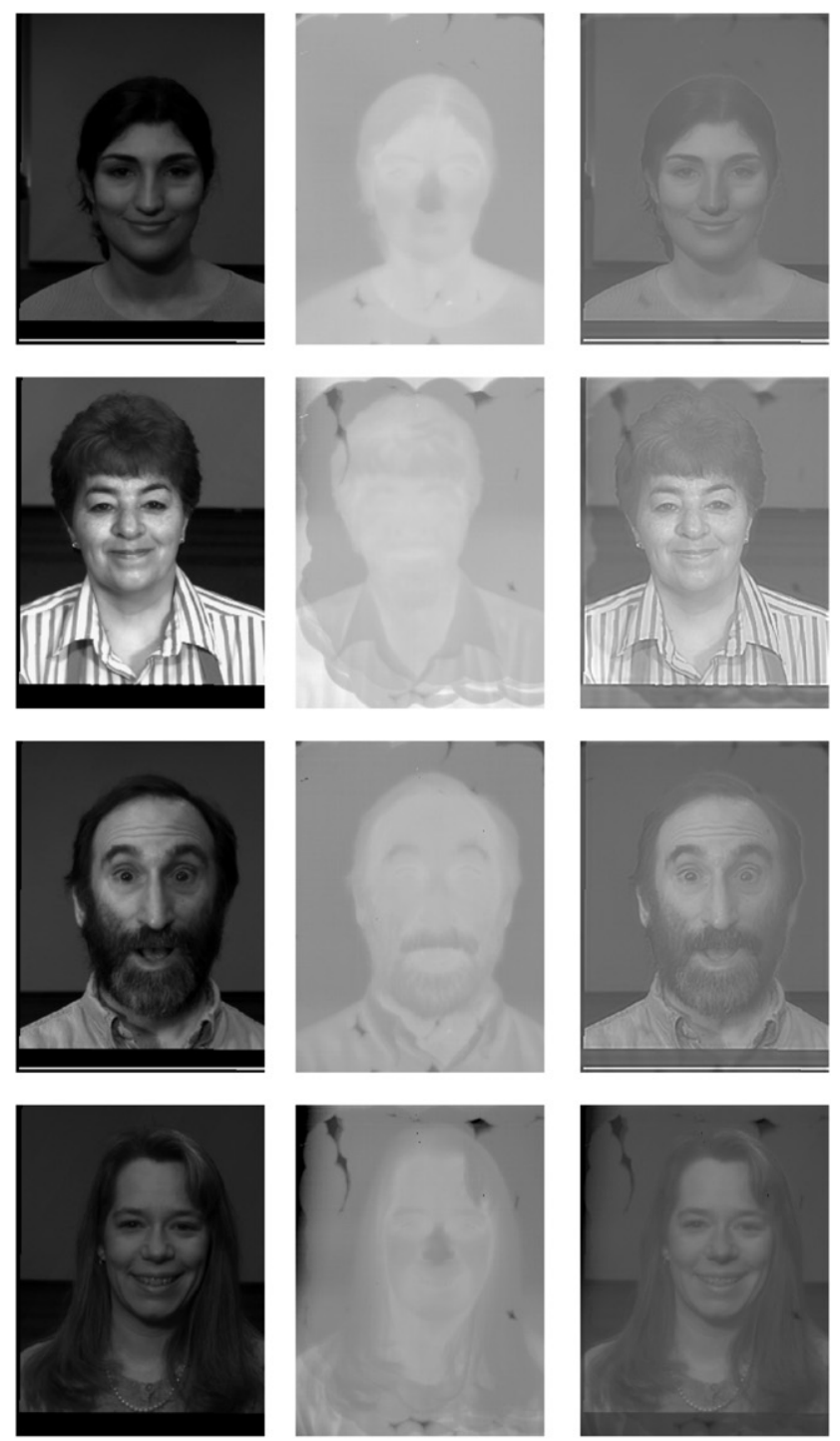

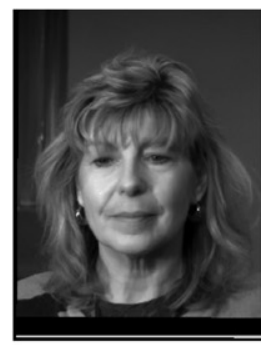

Visible Image

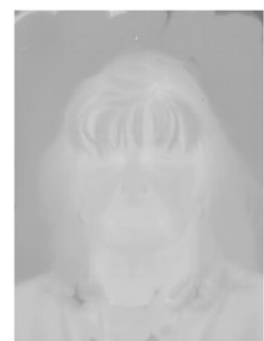

LWIR

Image

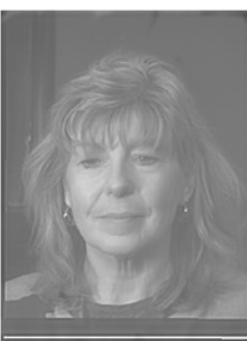

Fused Image
Fig. 9. Results of the proposed $2 v$-GSVM image fusion algorithm on the Equinox face database [19].

\subsection{Validation of integrated multilevel image and match score fusion algorithm}

In previous experiments, we have established that image fusion using $2 v$-GSVM improves the verification performance. Also at match score level, the fusion using DSm theory improves the verification accuracy even when the multispectral 
Table 3

Verification performance of the proposed DSm and existing match score fusion algorithms at $0.01 \%$ FAR

\begin{tabular}{|c|c|c|c|c|c|}
\hline \multirow[t]{2}{*}{ Face database } & \multicolumn{5}{|l|}{ Verification accuracy (\%) } \\
\hline & Product rule fusion [36] & Sum rule fusion [36] & SVM fusion [37] & DST fusion [39] & Proposed DSm fusion \\
\hline Notre Dame & 95.07 & 97.12 & 97.46 & 97.58 & 98.82 \\
\hline Equinox & 93.20 & 94.33 & 95.05 & 96.51 & 98.08 \\
\hline
\end{tabular}

a

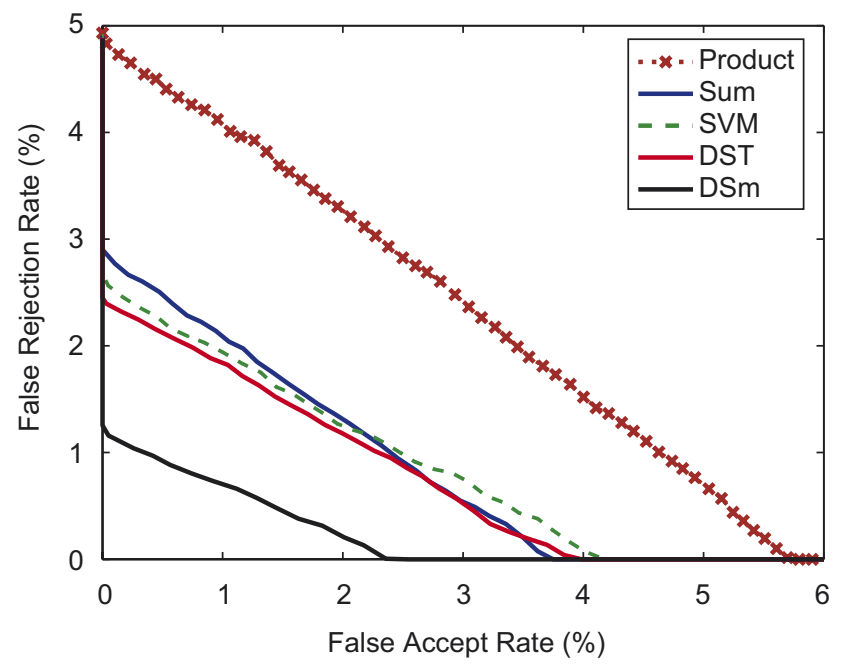

b

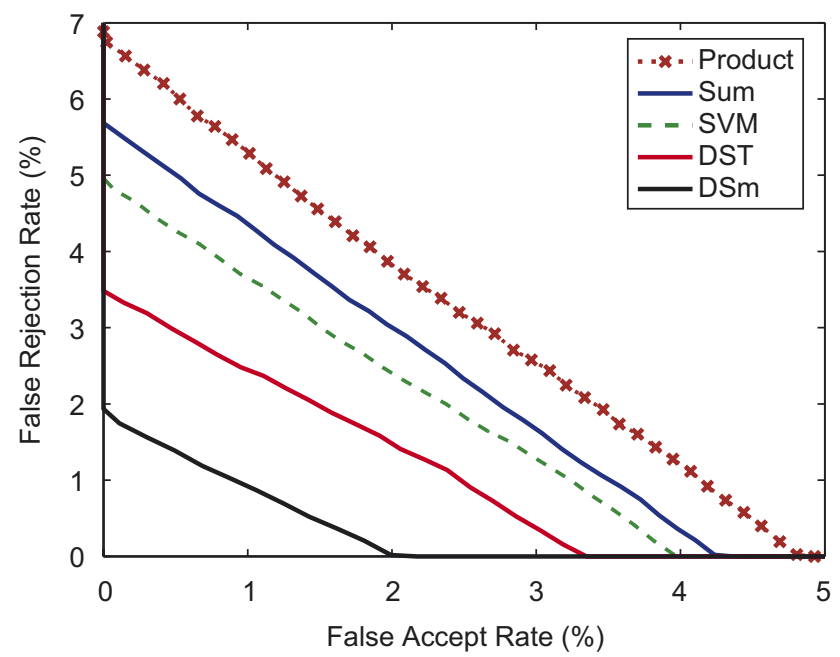

Fig. 10. ROC plots of the proposed DSm match score fusion and existing match score fusion algorithms on the (a) Notre Dame face database [18] (b) Equinox face database [19].

images are not fused. In this section, we integrate both image fusion and match score fusion algorithms as described in Section 5 to evaluate the face verification performance. The validation results of the integrated multilevel fusion algorithm are summarized in Table 4. The integrated fusion algorithm yields $99.91 \%$ verification accuracy on the Notre Dame face database and $99.54 \%$ on the Equinox face database. The results also show that the integration of fusion algorithms further improves the verification performance by at least $1.08 \%$ compared to only image fusion or only match score fusion. High verification accuracies ( $>99.5 \%$ ) on the Notre Dame and Equinox face databases show the robustness of the proposed integrated fusion algorithm to the variations in illumination, expression, and occlusion due to glasses. The computational time of the proposed integrated image and match score fusion algorithms including image registration, feature extraction and matching is $4.3 \mathrm{~s}$ on a $\mathrm{P}-\mathrm{IV}, 3.2 \mathrm{GHz}$ computer under MATLAB environment.

\subsection{Statistical evaluation of proposed image fusion and match score fusion algorithms}

The performance of a biometric system greatly depends on the database size and the images present in the database [46]. It cannot be represented completely by ROC plots and verification accuracy. To systematically evaluate the performance, Bengio and Marièthoz [47] have proposed statistical test using HTER and confidence intervals [47]. In this section, we perform statistical evaluation of the proposed image fusion and match score fusion algorithms using HTER. HTER is defined as,

$H T E R=\frac{F A R+F R R}{2}$.

Confidence intervals are computed around HTER as HTER $\pm \sigma$. $Z_{\alpha / 2} . \quad \sigma$ and $Z_{\alpha / 2}$ are computed using Eqs. (30) and (31) [47]

$\sigma=\sqrt{\frac{F A R(1-F A R)}{4 \cdot N I}+\frac{F R R(1-F R R)}{4 \cdot N G}}$,

$Z_{\alpha / 2}= \begin{cases}1.645 & \text { for } 90 \% C I \\ 1.960 & \text { for } 95 \% C I \\ 2.576 & \text { for } 99 \% C I\end{cases}$

$N G$ is the total number of genuine scores and $N I$ is the total number of impostor scores.

Table 5 summarizes the results of this statistical evaluation using false accept and false reject rates. We have computed these statistical values at $0.01 \%$ FAR. This statistical test shows that on a database similar to the Notre Dame with any number of classes, HTER of the proposed integrated fusion algorithm will lie between $0.05 \pm 0.06$ with $95 \%$ confidence. Similar results have been obtained with the Equinox face database. 
Table 4

Verification performance of the proposed integration of image fusion and match score fusion algorithms at $0.01 \%$ FAR

\begin{tabular}{|c|c|c|c|c|}
\hline \multirow[t]{3}{*}{ Face database } & \multicolumn{4}{|c|}{ Verification accuracy (\%) } \\
\hline & \multicolumn{2}{|c|}{ Proposed $2 v$-GSVM image fusion } & \multirow[t]{2}{*}{ Proposed DSm match score fusion } & \multirow[t]{2}{*}{ Integrated image and match score fusion } \\
\hline & 2D log polar Gabor & Local binary pattern & & \\
\hline Notre Dame & 95.85 & 94.80 & 98.82 & 99.91 \\
\hline Equinox & 94.98 & 94.71 & 98.08 & 99.54 \\
\hline
\end{tabular}

Table 5

Confidence interval around HTER of the proposed 2v-GSVM image fusion, DSm match score fusion, and integrated multilevel fusion algorithms

\begin{tabular}{|c|c|c|c|c|c|}
\hline \multirow[t]{2}{*}{ Face database } & \multirow[t]{2}{*}{ Fusion algorithms } & \multirow[t]{2}{*}{ HTER (\%) } & \multicolumn{3}{|c|}{ Confidence interval $(\%)$ around HTER for } \\
\hline & & & $90 \%$ & $95 \%$ & $99 \%$ \\
\hline \multirow[t]{4}{*}{ Notre Dame } & Image fusion with 2D log polar Gabor & 2.08 & 0.68 & 0.81 & 1.07 \\
\hline & Image fusion with local binary pattern & 2.61 & 0.76 & 0.91 & 1.19 \\
\hline & Match score fusion & 0.59 & 0.37 & 0.44 & 0.58 \\
\hline & Integrated image fusion and match score fusion & 0.05 & 0.10 & 0.12 & 0.16 \\
\hline \multirow[t]{4}{*}{ Equinox } & Image fusion with 2D log polar Gabor & 2.52 & 0.26 & 0.31 & 0.41 \\
\hline & Image fusion with local binary pattern & 2.65 & 0.27 & 0.32 & 0.42 \\
\hline & Match score fusion & 0.97 & 0.17 & 0.20 & 0.26 \\
\hline & Integrated image fusion and match score fusion & 0.24 & 0.08 & 0.10 & 0.13 \\
\hline
\end{tabular}

\section{Conclusion}

Visible and long wave infrared images provide complementary properties which can be combined to improve the performance of face recognition. In this paper, we proposed image fusion and match score fusion algorithms to fuse information obtained from multispectral face images. We first apply mutual information based registration algorithm to register multispectral face images and then fuse the images using the proposed $2 v$-granular support vector machine. The fused image contains the properties of both visible and long wave infrared images and can efficiently be used for face recognition. We next proposed the DSm match score fusion algorithm to fuse match scores generated from multiple classifiers. These match scores can be generated by applying multiple classifiers on one image or by applying classifiers on multispectral face images. The proposed match score fusion algorithm is based on the theory of evidence and performs efficiently even when visible and long wave infrared images provide conflicting decisions. The proposed image fusion and match score fusion algorithms are then integrated to further improve the face recognition performance. We validated the performance of the proposed image fusion algorithm, match score fusion algorithm, and the integrated image fusion and match score fusion algorithms using the Notre Dame and Equinox face databases. Experimental results show that the proposed image fusion and match score fusion algorithms outperform existing fusion algorithms. Results and statistical evaluation further show that the proposed integrated image and match score fusion algorithm yield best performance among all the proposed and existing fusion algorithms.

\section{Acknowledgment}

The authors would like to thank CVRL University of Notre Dame and Equinox Corporation for providing the face databases used in this research.

\section{References}

[1] W. Zhao, R. Chellappa, A. Rosenfeld, P.J. Phillips, Face recognition: a literature survey, ACM Comput. Surv. 12 (4) (2003) 399-458.

[2] X. Chen, P. Flynn, K. Bowyer, IR and visible light face recognition, Comput. Vision Image Understanding 99 (3) (2005) 332-358.

[3] S. Kong, J. Heo, B. Abidi, J. Paik, M. Abidi, Recent advances in visual and infrared face recognition-a review, J. Comput. Vision Image Understanding 97 (1) (2005) 103-135.

[4] S.G. Kong, J. Heo, F. Boughorbel, Y. Zheng, B.R. Abidi, A. Koschan, M. Yi, M.A. Abidi, Multiscale fusion of visible and thermal IR images for illumination-invariant face recognition, Int. J. Comput. Vision 71 (2) (2007) 215-233.

[5] F. Prokoski, History, current status, and future of infrared identification, in: Proceedings of IEEE Workshop on Computer Vision beyond the Visible Spectrum: Methods and Applications, 2000, pp. 5-14.

[6] D. Socolinsky, A. Selinger, J. Neuheisel, Face recognition with visible and thermal infrared imagery, J. Comput. Vision Image Understanding 91 (2003) 72-114.

[7] J. Wilder, J. Phillips, C. Jiang, S. Wiener, Comparison of visible and infra-red imagery for face recognition, in: Proceedings of International Conference on Automatic Face and Gesture Recognition, 1996, pp. $182-187$.

[8] A. Gyaourova, G. Bebis, I. Pavlidis, Fusion of infrared and visible images for face recognition, Lecture Notes in Computer Science, vol. 3024, Springer, Berlin, 2004 pp. 456-468.

[9] J. Heo, S. Kong, B. Abidi, M. Abidi, Fusion of visual and thermal signatures with eyeglass removal for robust face recognition, in: Proceedings of IEEE Workshop on Object Tracking and Classification 
Beyond the Visible Spectrum in Conjunction with CVPR, 2004, pp. 94-99.

[10] R. Singh, M. Vatsa, A. Noore, Hierarchical fusion of multi-spectral face images for improved recognition performance, Inf. Fusion (2007), in press, doi:10.1016/j.inffus.2006.06.002.

[11] S. Singh, A. Gyaourova, G. Bebis, I. Pavlidis, Infrared and visible image fusion for face recognition, in: Proceedings of SPIE Defense and Security Symposium (Biometric Technology for Human Identification), vol. 5404, 2004, pp. 585-596.

[12] R. Singh, M. Vatsa, A. Noore, Intelligent biometric information fusion using support vector machine, in: M. Nachtegael, D. Van der Weken, E.E. Kerre, W. Philips (Eds.), Soft Computing in Image Processing: Recent Advances, Springer, Berlin, 2006, pp. 327-350, (Chapter 12).

[13] R. Singh, M. Vatsa, A. Noore, Face recognition with disguise and single gallery images, Image Vision Comput. (2007), in press, doi:10.1016/j.imavis.2007.06.010.

[14] T. Ahonen, A. Hadid, M. Pietikäinen, Face description with local binary patterns: application to face recognition, IEEE Trans. Pattern Anal. Mach. Intell. 28 (12) (2006) 2037-2041.

[15] J. Dezert, Foundations for a new theory of a plausible and paradoxical reasoning, Inf. Secur. J. 9 (2002) 13-57.

[16] F. Smarandache, J. Dezert, Advances and Applications of DSmT for Information Fusion, American Research Press, 2004.

[17] X. Chen, P.J. Flynn, K.W. Bowyer, Visible-light and infrared face recognition, in: Proceedings of ACM Workshop on Multimodal User Authentication, 2003, pp. 48-55.

[18] P.J. Flynn, K.W. Bowyer, P.J. Phillips, Assessment of time dependency in face recognition: an initial study, in: Proceedings of International Conference on Audio and Video-Based Biometric Person Authentication, 2003, pp. 44-51.

[19] Equinox face database: 〈http://www.equinoxsensors.com/products/ HID.html>.

[20] V.N. Vapnik, The Nature of Statistical Learning Theory, Springer, Berlin, 1995.

[21] P.-H. Chen, C.-J. Lin, B. Schölkopf, A tutorial on v-support vector machines, Appl. Stochastic Models Bus. Ind. 21 (2005) 111-136.

[22] H.G. Chew, C.C. Lim, R.E. Bogner, An implementation of training dual$v$ support vector machines, in: Qi, Teo, Yang (Eds.), Optimization and Control with Applications, Kluwer, Dordrecht, 2004.

[23] Y.C. Tang, B. Jin, Y.-Q. Zhang, Granular support vector machines with association rules mining for protein homology prediction, Artif. Intell. Med. 35 (1-2) (2005) 121-134 (special issue on Computational Intelligence Techniques in Bioinformatics).

[24] Y.C. Tang, Y.-Q. Zhang, Granular support vector machines with data cleaning for fast and accurate biomedical binary classification, in: Proceedings of International Conference on Granular Computing, 2005, pp. 262-265.

[25] Y.C. Tang, B. Jin, Y.-Q. Zhang, H. Fang, B. Wang, Granular support vector machines using linear decision hyperplanes for fast medical binary classification, in: Proceedings of International Conference on Fuzzy Systems, 2005, pp. 138-142.

[26] Y.C. Tang, Y.-Q. Zhang, Granular SVM with repetitive undersampling for highly imbalanced protein homology prediction, in: Proceedings of International Conference on Granular Computing, 2006, pp. 457-461.

[27] A. Bargiela, W. Pedrycz, Granular Computing: An Introduction, Kluwer International Series in Engineering and Computer Science, 2002.

[28] A. Bargiela, W. Pedrycz, The roots of granular computing, in: Proceedings of IEEE International Conference on Granular Computing, 2006, pp. 806-809.
[29] Y.H. Chen, Y.Y. Yao, Multiview intelligent data analysis based on granular computing, in: Proceedings of IEEE International Conference on Granular Computing, 2006, pp. 281-286.

[30] Y.Y. Yao, Perspectives of granular computing, in: Proceedings of IEEE International Conference on Granular Computing, vol. 1, 2005 , pp. 85-90.

[31] G. Bebis, A. Gyaourova, S. Singh, I. Pavlidis, Face recognition by fusing thermal infrared and visible imagery, Image Vision Comput. 24 (7) (2006) 727-742.

[32] F. Maes, A. Collignon, D. Vandermeulen, G. Marchal, P. Suetens, Multimodality image registration by maximization of mutual information, IEEE Trans. Med. Imaging 16 (2) (1997) 187-198.

[33] J.P. Pluim, J.B.A. Maintz, M.A. Viergever, Mutual information-based registration of medical images: a survey, IEEE Trans. Med. Imaging 22 (8) (2003) 986-1004.

[34] D. Hill, C. Studholme, D. Hawkes, Voxel similarity measures for automated image registration, in: Proceedings of the Third SPIE Conference on Visualization in Biomedical Computing, 1994, pp. 205-216.

[35] M. Antonini, M. Barlaud, P. Mathieu, I. Daubechies, Image coding using the wavelet transform, IEEE Trans. Image Process. 1 (2) (1992) 205-220

[36] A. Ross, A.K. Jain, Information fusion in biometrics, Pattern Recognition Lett. 24 (13) (2003) 2115-2125.

[37] J.F. Aguilar, J.O. Garcia, J.G. Rodriguez, J. Bigun, Kernel-based multimodal biometric verification using quality signals, in: Proceedings of SPIE Biometric Technology for Human Identification, vol. 5404, 2004, pp. 544-554.

[38] G. Shafer, A Mathematical Theory of Evidence, Princeton University Press, Princeton, NJ, 1976.

[39] R. Singh, M. Vatsa, A. Noore, S.K. Singh, Dempster Shafer theory based classifier fusion for improved fingerprint verification performance, in: Proceedings of Indian Conference on Computer Vision, Graphics and Image Processing, Lecture Notes in Computer Science, vol. 4338, Springer, Berlin, 2006, pp. 941-949.

[40] L. Zadeh, On the validity of Dempster's rule of combination, Memo M 79/24, University of California, Berkeley, 1979.

[41] L. Zadeh, Review of mathematical theory of evidence by Glenn Shafer, AI Mag. 5 (3) (1984) 81-83.

[42] L. Zadeh, A simple view of the Dempster-Shafer theory of evidence and its implication for the rule of combination, AI Mag. 7 (2) (1986) 85-90.

[43] D. Dubois, H. Prade, On the unicity of Dempster rule of combination, Int. J. Intell. Systems 1 (1986) 133-142.

[44] F. Voorbraak, On the justification of Dempster's rule of combination, Artif. Intell. 48 (1991) 171-197.

[45] S.K. Singh, D.S. Chauhan, M. Vatsa, R. Singh, A robust skin color based face detection algorithm, Tamkang J. Sci. Eng. 6 (4) (2003) 227-234.

[46] R.M. Bolle, N.K. Ratha, S. Pankanti, Performance evaluation in 1:1 biometric engines, in: Proceedings of Sinobiometrics, 2004, pp. 27-46.

[47] S. Bengio, J. Marièthoz, A statistical significance test for person authentication, in: Proceedings of Odyssey: The Speaker and Language Recognition Workshop, 2004, pp. 237-244.

\footnotetext{
About the Author-RICHA SINGH is a graduate research assistant in the Lane Department of Computer Science and Electrical Engineering at West Virginia University. She is currently pursuing her Doctoral degree in Computer Science. She had been actively involved in the development of a multimodal biometric system which includes face, fingerprint, signature, and iris recognition at Indian Institute of Technology Kanpur, India, from July 2002 to July 2004. Her current areas of interest are pattern recognition, image processing, machine learning, granular computing, biometric authentication, and data fusion. Richa has more than 55 publications in refereed journals, book chapters, and conferences, and has received three best paper awards. She is a member of the IEEE, Computer Society, and SPIE. She is also a member of Phi Kappa Phi, Upsilon Pi Epsilon, and Eta Kappa Nu honor societies.
} 
About the Author-MAYANK VATSA is a graduate research assistant in the Lane Department of Computer Science and Electrical Engineering at West Virginia University. He is currently pursuing his Doctoral degree in Computer Science. He had been actively involved in the development of a multimodal biometric system which includes face, fingerprint, signature, and iris recognition at Indian Institute of Technology Kanpur, India, from July 2002 to July 2004. His current areas of interest are pattern recognition, image processing, uncertainty principles, biometric authentication, watermarking, and information fusion. Mayank has more than 55 publications in refereed journals, book chapters, and conferences. He has received three best paper awards. He is a member of the IEEE, Computer Society, and SPIE. He is also a member of Phi Kappa Phi, Sigma Xi, Upsilon Pi Epsilon, and Eta Kappa Nu honor societies.

About the Author-AFZEL NOORE received his Ph.D. in Electrical Engineering from West Virginia University. He worked as a digital design engineer at Philips India. From 1996 to 2003, Dr. Noore served as the associate dean for Academic Affairs and Special Assistant to the Dean in the College of Engineering and Mineral Resources at West Virginia University. He is a professor in the Lane Department of Computer Science and Electrical Engineering. His research interests include computational intelligence, biometrics, software reliability modeling, machine learning, hardware description languages, and quantum computing. His research has been funded by NASA, NSF, Westinghouse, GE, Electric Power Research Institute, the US Department of Energy, and the US Department of Justice. Dr. Noore has over 80 publications in refereed journals, book chapters, and conferences. He has received three best paper awards. Dr. Noore is a member of the IEEE and serves in the editorial boards of Recent Patents on Engineering, Open Nanoscience Reviews, Open Nanoscience Letters, and the Open Nanoscience Journal. He is a member of Phi Kappa Phi, Sigma Xi, Eta Kappa Nu, and Tau Beta Pi honor societies. 